\title{
Minimal-dose computed tomography is superior to chest $x$-ray for the follow-up and treatment of patients with resected lung cancer
}

\author{
Waël C. Hanna, MBA, MD, ${ }^{a}$ Narinder S. Paul, MD, ${ }^{\mathrm{b}}$ Gail E. Darling, MD, ${ }^{\mathrm{a}}$ Hadas Moshonov, PhD, ${ }^{\mathrm{b}, \mathrm{c}}$ \\ Frances Allison, MSc, ${ }^{a}$ Thomas K. Waddell, MD, PhD, ${ }^{a}$ Marcelo Cypel, MD, ${ }^{a}$ Marc E. de Perrot, MD, \\ Kazuhiro Yasufuku, MD, PhD, ${ }^{a}$ Shaf Keshavjee, MSc, MD, ${ }^{a}$ and Andrew F. Pierre, MSc, $\mathrm{MD}^{\mathrm{a}}$
}

\begin{abstract}
Objectives: A minimal-dose computed tomography scan of the thorax (MnDCT) delivers a radiation dose comparable with a chest X-ray (CXR). We hypothesized that in patients with completely resected lung cancer, surveillance with MnDCT, when compared with CXR, leads to earlier detection and a higher rate of treatment of new or recurrent lung cancer.
\end{abstract}

\begin{abstract}
Methods: After lung cancer resection, patients prospectively were enrolled for surveillance with MnDCT and CXR at $3,6,12,18,24,36,48$, and 60 months. Images were interpreted by different blinded radiologists. When new or recurrent cancer was suspected, standard-dose $\mathrm{CT}$ and/or a tissue biopsy were performed for confirmation.
\end{abstract}

\begin{abstract}
Results: Between 2007 and 2012, 271 patients were included and 1137 pairs of CXR and MnDCT were analyzed. MnDCT was more sensitive $(94 \%$ vs $21 \% ; P<.0001)$ and had a higher negative predictive value (99\% vs $96 \% ; P=.007)$ than CXR for the diagnosis of new or recurrent lung cancer. The prevalence of new or recurrent lung cancer was $23.2 \%$ (63 of 271), of whom 78\% (49 of 63) had asymptomatic disease. The majority of asymptomatic patients $(75 \%$; 37 of 49$)$ were treated with curative intent and had a median survival of 69 months. The remainder of patients received palliative treatment $(24 \% ; 12$ of 49$)$ and had a median survival of 25 months $(P<.0001)$.
\end{abstract}

Conclusions: After curative resection of lung cancer, MnDCT is superior to CXR for the detection of new or recurrent lung cancer. The majority of new or recurrent cancer was detected by MnDCT at an asymptomatic phase, allowing for curative treatment, leading to a long survival. ( $\mathrm{J}$ Thorac Cardiovasc Surg 2014;147:30-5)

\section{Earn CME credits at \\ http://cme.ctsnetjournals.org}

Survivors of lung cancer surgery are among the highest-risk patients for developing another lung cancer, either a new metachronous primary tumor $(1 \%-2 \%$ per year risk $)$ or recurrent disease (10\%-38\% overall risk). ${ }^{1,2}$ Despite this, there is no clear consensus on the follow-up evaluation of patients after curative surgical resection of lung cancer. ${ }^{3}$ Although surveillance with periodic chest $\mathrm{x}$-ray (CXR) is indicated in most published guidelines, ${ }^{4}$ recent recommendations for the use of a routine thoracic computed

From the Division of Thoracic Surgery, ${ }^{\mathrm{a}}$ Department of Radiology, ${ }^{\mathrm{b}}$ and Division of Biostatistics, ${ }^{\mathrm{c}}$ University of Toronto, Toronto, Ontario, Canada.

Disclosures: Authors have nothing to disclose with regard to commercial support.

Read at the 93rd Annual Meeting of The American Association for Thoracic Surgery, Minneapolis, Minnesota, May 4-8, 2013.

Received for publication May 4, 2013; revisions received Aug 17, 2013; accepted for publication Aug 29, 2013; available ahead of print Oct 21, 2013.

Address for reprints: Andrew F. Pierre, MD, MSc, FRCSC, Toronto General Hospital, 200 Elizabeth St, 9N-950 (E-mail: andrew.pierre@uhn.ca).

$0022-5223 / \$ 36.00$

Copyright $($ C 2014 by The American Association for Thoracic Surgery

http://dx.doi.org/10.1016/j.jtcvs.2013.08.060 tomography (CT) scan are starting to emerge. ${ }^{5}$ We hypothesized that, after lung cancer resection, surveillance with minimal-dose CT of the chest (MnDCT) leads to earlier detection and a higher rate of treatment of new or recurrent lung cancer, when compared with CXR.

\section{MATERIALS AND METHODS}

After Institutional Review Board approval, patients who had undergone lung cancer resection were invited to participate in this prospective study at the time of their first postoperative visit. Consenting patients were seen in the clinic every 3 months for the first 2 years after surgery, then every 6 months until 5 years after surgery, and then yearly thereafter. Each patient underwent surveillance with both MnDCT of the thorax and CXR at 3, 6, 12, 18, 24, 36, 48, and 60 months after surgery. Patients remained on study protocol until they were diagnosed with new or recurrent lung cancer, or until they had reached 5 years without evidence of disease. All MnDCT scans were acquired with the use of 64-row multidetector scanners (Aquilion 64; Toshiba Medical Systems, Ottawara, Japan) using a tube potential of $120 \mathrm{kVp}$, a fixed tube current of $10 \mathrm{~mA}$ with a gantry rotation of $500 \mathrm{~ms}$, resulting in an average effective dose of $0.2 \mathrm{mSv}$. The average effective dose with standard-dose chest CT varies widely but is approximately $8 \mathrm{mSv}{ }^{6}$ Two-view digital chest radiography was performed at an average effective dose of $0.16 \mathrm{mSv}$. All CXRs were interpreted by subspecialty board certified thoracic radiologists who were blinded to the MnDCT. The MnDCT images were interpreted by subspecialty board certified chest radiologists who were blinded to the CXR. The images and interpretation reports of both tests then were reviewed by the thoracic surgeon. A test was termed "positive" when it was 


\section{Abbreviations and Acronyms \\ CT = computed tomography \\ $\mathrm{CXR}=$ chest $\mathrm{x}$-ray \\ $\mathrm{MnDCT}=$ minimal-dose computed tomography}

explicitly mentioned in the official interpretation report that the study was suspicious for recurrent or new lung cancer. Common findings that led to positive studies were noncalcified nodules, intrathoracic lymphadenopathy, or pleural effusion. Whenever a positive study was reported, further confirmatory investigations such as standard-dose high-resolution thoracic CT and/or tissue biopsy were ordered at the discretion of the treating surgeon. Patients with false-positive screening studies returned to surveillance per study protocol. Patients with a confirmed diagnosis of cancer were restaged and treated with either curative or palliative intent per the recommendation of a multidisciplinary tumor board.

The gold standard for calculating sensitivity was the pathologic specimen in patients who underwent biopsy or surgery, and the administration of lung cancer treatment in patients who did not achieve a pathologic diagnosis. When the diagnosis of lung cancer was confirmed, all prior MnDCT scans were reviewed to determine whether they were false negatives or true positives.

Statistical analysis was performed using SPSS software version 20 (SPSS Inc, Chicago, Ill). Continuous variables were described using mean and standard deviation and categoric variables using frequency and percentage. Logistic regression analysis was used to compare diagnostic performance measures between the 2 imaging modalities and generalized estimating equations were used to adjust for correlations of repeated measures. Kaplan-Meier survival analysis was performed; survival curves and estimates of median survival time are provided. The log-rank test was used for comparison of survival functions. The level of significance was set at a $P$ value less than .05 .

\section{RESULTS}

\section{Demographics}

Between 2007 and 2012, 311 patients were enrolled in the study and 271 patients were analyzed. Forty patients were excluded for the following reasons: voluntary withdrawal (14 of 40), loss to follow-up evaluation (12 of 40), death before the 3-month visit ( 8 of 40), recurrence before the 3 -month visit (4 of 40), and development of non-lung cancer requiring imaging and treatment ( 2 of 40 ). The mean age was $66.6 \pm 9.5$ years (range, $40-90$ year), $53.5 \%$ of patients were women, and the majority $(79.7 \%)$ were smokers. Pathologic staging of disease was performed according to the 7th edition of the American Joint Committee on Cancer/International Union Against Cancer (Union Internationale Contre Cancer) TNM staging manual. ${ }^{7}$ Stage I disease was found in $79.7 \%$ of patients, stage II in $12.5 \%$ of patients, stage III in $6.6 \%$ of patients, and stage IV in $1.1 \%$ of patients. The majority of patients had a lobar resection $(77.9 \%)$, and $18.1 \%$ had a sublobar resection, $1.8 \%$ had a bilobectomy, and $2.2 \%$ had a pneumonectomy. Video-assisted thoracoscopic surgery was used in $36.5 \%$ of cases. Chemotherapy and radiation therapy were used in $21.4 \%$ and $12.5 \%$ of patients, respectively.

\section{Diagnostic Performance of MnDCT Versus CXR}

A total of 1137 pairs of MnDCT and CXR were analyzed. The sensitivity of MnDCT for the diagnosis of new or recurrent lung cancer after surgical resection was $94.2 \%$ (95\% CI, 84.1-98.8) versus $21.2 \%$ for CXR $(95 \% \mathrm{CI}$, $11.1-34.72 ; P<.0001)$. There were no cancers diagnosed on CXR that were not detected on MnDCT. When no signs of lung cancer were detected, the negative predictive value of MnDCT was $99.7 \%$ (range, $99.0 \%-99.9 \%$ ) versus $96.2 \%$ (range, $94.9 \%-97.2 \%$ ) for CXR $(P=.007)$. MnDCT has a specificity of $86 \%$ (range, $83.7 \%-88.1 \%$ ) and a positive predictive value of $25.1 \%$ (range, $19.2 \%$ $31.8 \%$ ) for the diagnosis of new or recurrent lung cancer, versus $99.9 \%$ (range, $99.5 \%-99.9 \%$ ) and $91.7 \%$ (range, 61.5\%-99.8\%), respectively, for CXR $(P<.0001)$.

\section{Patterns of Further Investigation After Positive MnDCT}

Positive MnDCT screening studies led to further investigation by the surgeon in only $54.4 \%$ of cases. Further tests consisted of standard-dose CT of the chest in $79.1 \%$ of cases and invasive procedures (bronchoscopy or CT-guided biopsy) in only $20.9 \%$. Further investigations were not pursued in $45.6 \%$ of positive MnDCT studies, in which the surgeon used clinical judgment to classify the result as a false positive. This judgment was correct in $81 \%$ of cases, as evidenced by further follow-up evaluation.

\section{Patterns of Recurrent or New Lung Cancer}

New or recurrent lung cancer was confirmed in $23.2 \%$ (63 of 271) of patients. Among multiple risk factors analyzed in a multivariate logistic regression model, bilobar resection and stage III disease were identified as predictors of recurrence or new lung cancer, whereas multiple nodules and male sex showed a trend toward statistical significance (Table 1). The majority of patients with cancer $(77.8 \% ; 49$ of 63$)$ presented with asymptomatic disease that was diagnosed only by imaging (Figure 1). Two thirds of patients (67.3\%; 32 of 49) were diagnosed on MnDCT within the first year of surveillance, and $26.5 \%$ (13 of 49) were diagnosed within the second year. No asymptomatic patients were diagnosed in the fourth and fifth year (Figure 2). Symptomatic disease was diagnosed in $22.2 \%$ (14 of 63 ). At the present time, $52.4 \%$ (142 of 271) of patients remain disease free with a median follow-up period of 36 months, and $24.3 \%$ (66 of 271) are disease free at 5 years (Figure 1).

\section{Patterns of Treatment and Survival}

The majority of patients with asymptomatic new or recurrent lung cancer $(75.5 \% ; 37$ of 49$)$ were found to be candidates for curative treatment with further surgery or radiation. The median survival for patients who were treated with curative intent was 69 months (range, 12-76 months) 
TABLE 1. The predictors of recurrent or new lung cancer after surgical resection

\begin{tabular}{lccc}
\hline \multicolumn{1}{c}{ Risk factors } & $\begin{array}{c}\text { Odds } \\
\text { ratio }\end{array}$ & $\begin{array}{c}\mathbf{9 5} \% \text { Confidence } \\
\text { interval }\end{array}$ & $\begin{array}{c}\boldsymbol{P} \\
\text { value }\end{array}$ \\
\hline Bilobar resection & 6.813 & $1.108-41.89$ & .048 \\
Stage III & 2.962 & $1.157-7.584$ & .036 \\
Multiple pulmonary nodules & 2.205 & $0.982-4.951$ & .05 \\
Male sex & 1.845 & $0.995-3.423$ & .05 \\
Age & - & - & .07 \\
Chemotherapy treatment & - & - & .43 \\
Radiotherapy treatment & - & - & .22 \\
History of other cancer & - & - & .12 \\
Continued to smoke & - & - & .60 \\
\hline
\end{tabular}

from the initial surgery (Figure 1). On the contrary, all the patients who presented with symptomatic disease (14 of 14) were found to be ineligible for curative intent therapy. These patients were referred to treatment with palliative intent and had a median survival of 15 months (range, 7-63 months) from the initial surgery. In addition, 24.5\% (12 of 49) of patients with asymptomatic disease also were not candidates for further curative treatment and therefore were treated with palliative intent, with a median survival of 25 months (range, 6-48 months). The survival of patients who were referred to palliative treatment was significantly shorter than the survival of patients treated with curative intent $(P<.0001)$ (Figure 3$)$.

\section{DISCUSSION}

Screening trials have shown that CT is superior to CXR in detecting early stage lung cancer. However, survivors of lung cancer were excluded from these studies and it is still unknown how CT compares with CXR in postoperative surveillance. ${ }^{8-16}$ In this prospective blinded study, we compared MnDCT with CXR in the follow-up period after lung cancer resection. The trial was designed to minimize bias by using the same patient population for the intervention (MnDCT) and the control (CXR) arms. We show that MnDCT is vastly superior to CXR in the detection of new or recurrent lung cancer. More importantly, the advantage

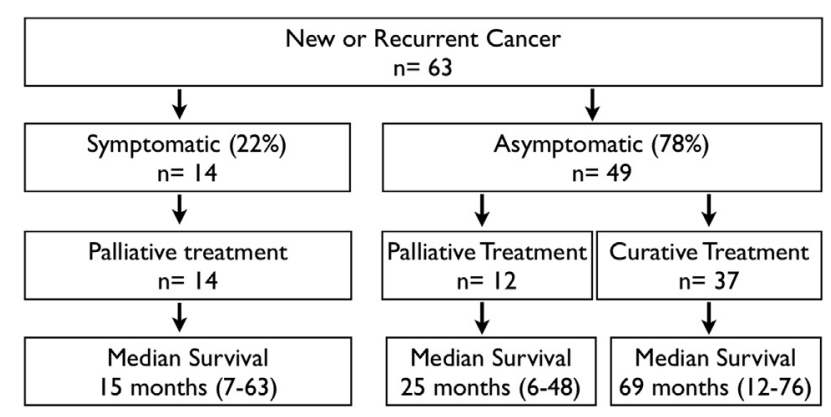

FIGURE 1. Clinical pathways of patients who were diagnosed with new or recurrent lung cancer during surveillance.

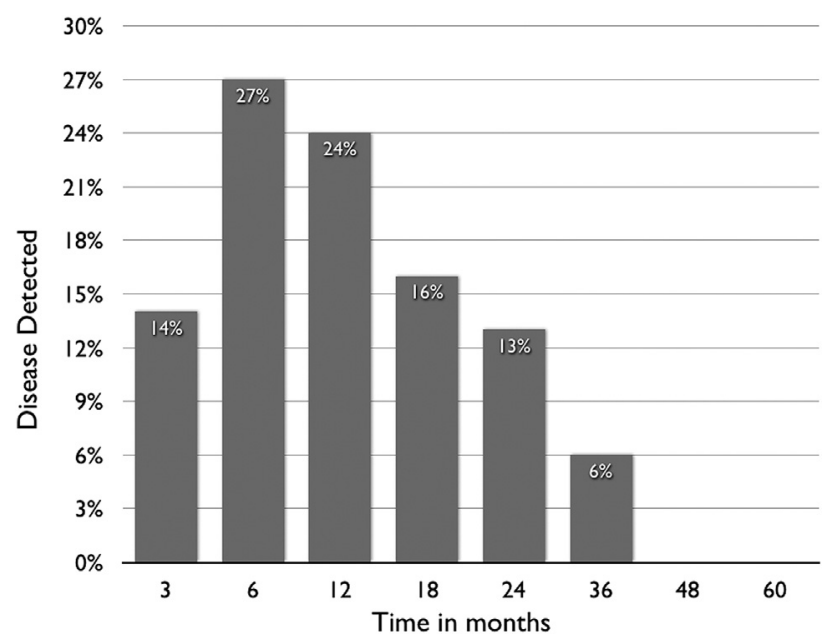

FIGURE 2. Distribution over time (in months) of the recurrences or new cancers diagnosed by MnDCT.

in sensitivity of MnDCT did not come at the cost of higher radiation exposure because the effective radiation dose $(0.2 \mathrm{mSv})$ was comparable with that of CXR $(0.16 \mathrm{mSv})$. However, high sensitivity comes at the expense of low positive predictive value, which has been documented in previous CT screening trials. ${ }^{15,17,18}$ Nevertheless, at our institution, surgeons were able to recognize false-positive results with higher accuracy than radiologists and only half of the positive MnDCT studies led to further investigation. This is consistent with previous studies on the surgeon's ability to use clinical judgment in interpreting an oversensitive screening test, and highlights the surgeon's active role in decreasing the potential cost and morbidity of overinvestigation. $^{17}$

Extrathoracic metastatic disease, especially to the brain, has been considered the most common site of recurrence in patients with resected lung cancer. ${ }^{19-21}$ In contrast, we have shown that with MnDCT, the majority of patients can be

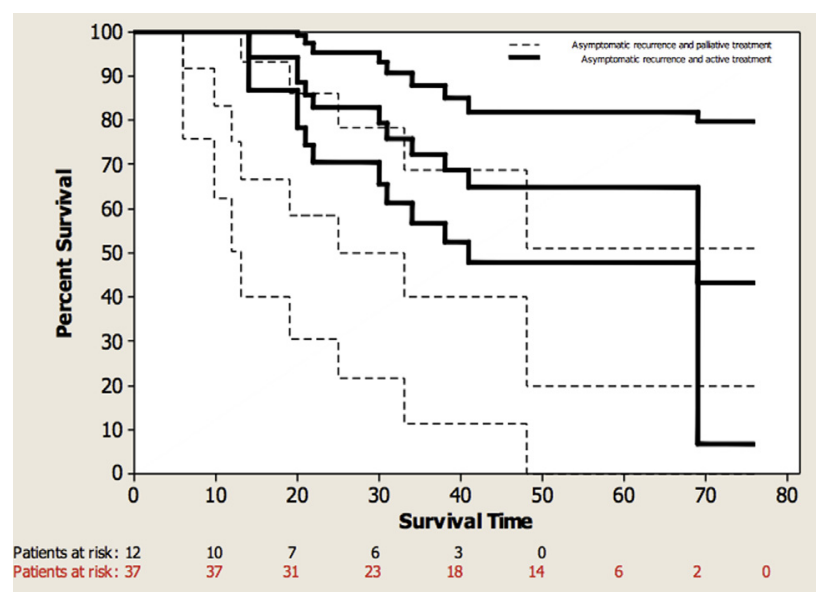

FIGURE 3. Survival in months since initial surgery. 
diagnosed at the intrathoracic and asymptomatic stage, within 2 years of surgery, when potentially curative intervention still is possible. Other investigators have shown that, in patients with previously resected lung cancer, curative surgery for a second lung cancer can be associated with a 5-year survival rate of $60 \% .^{22,23}$ In this study, the majority of asymptomatic patients were offered curative treatment that was associated with a median survival of more than 5 years, suggesting that efforts directed toward early intervention are associated with encouraging survival in a subset of patients with asymptomatic disease.

There were several limitations to this study. Although MnDCT can be performed in any setting, the radiologists at our hospital have extensive experience in interpreting low-dose and ultra-low-dose images, and this may not be generalizable to other institutions. MnDCT is unable to distinguish between new and recurrent lung cancer, a distinction that is important in terms of prognosis. ${ }^{18,24} \mathrm{In}$ addition, lead time and length time bias can falsely improve outcomes for asymptomatic patients detected early by MnDCT. Finally, because of small numbers and ongoing follow-up evaluation, this study may not be able to answer the question of whether MnDCT screening after lung cancer resection will improve survival in patients who are in remission.

\section{CONCLUSIONS}

MnDCT is superior to CXR for the detection of new or recurrent lung cancer after curative resection of a previous lung cancer. The majority of new or recurrent cancers was detected by MnDCT at an asymptomatic, intrathoracic stage, within 2 years of surgery. This allowed for the delivery of curative treatment in the majority of patients with asymptomatic cancer and was associated with long survival.

\section{References}

1. Haraguchi S, Koizumi K, Hirata T, Hirai K, Mikami I, Kubokura H, et al. Surgical treatment of metachronous nonsmall cell lung cancer. Ann Thorac Cardiovasc Surg. 2010;16:319-25.

2. Pepek JM, Chino JP, Marks LB, D’Amico TA, Yoo DS, Onaitis MW, et al. How well does the new lung cancer staging system predict for local/regional recurrence after surgery? A comparison of the TNM 6 and 7 systems. $J$ Thorac Oncol. 2011;6:757-61.

3. Rubins J, Unger M, Colice GL. Follow-up and surveillance of the lung cancer patient following curative intent therapy: ACCP Evidence-Based Clinical Practice Guideline (2nd edition). Chest. 2007;132(Suppl):355S-67S.

4. Calman L, Beaver K, Hind D, Lorigan P, Roberts C, Lloyd-Jones M. Survival benefits from follow-up of patients with lung cancer: a systematic review and meta-analysis. J Thorac Oncol. 2011;6:1993-2004.

5. Jaklitsch MT, Jacobson FL, Austin JHM, Field JK, Jett JR, Keshavjee S, et al. The American Association for Thoracic Surgery guidelines for lung cancer screening using low-dose computed tomography scans for lung cancer survivors and other high-risk groups. J Thorac Cardiovasc Surg. 2012;144:33-8.

6. Cagnon CH, Cody DD, McNitt-Gray MF, Seibert JA, Judy PF, Aberle DR. Description and implementation of a quality control program in an imaging-based clinical trial. Acad Radiol. 2006;13:1431-41.

7. Edge SB. American Joint Committee on Cancer: AJCC Cancer Staging Manual. New York: Springer; 2009.
8. Garg K, Keith RL, Byers T, Kelly K, Kerzner AL, Lynch DA, et al. Randomized controlled trial with low-dose spiral CT for lung cancer screening: feasibility study and preliminary results. Radiology. 2002;225:506-10.

9. Lopes Pegna A, Picozzi G, Mascalchi M, Maria Carozzi F, Carrozzi L, Comin C, et al. Design, recruitment and baseline results of the ITALUNG trial for lung cancer screening with low-dose CT. Lung Cancer. 2009;64:34-40.

10. Gohagan JK, Marcus PM, Fagerstrom RM, Pinsky PF, Kramer BS, Prorok PC, et al. Final results of the Lung Screening Study, a randomized feasibility study of spiral CT versus chest X-ray screening for lung cancer. Lung Cancer. 2005; 47:9-15.

11. Blanchon T, Bréchot J-M, Grenier PA, Ferretti GR, Lemarié E, Milleron B, et al. Baseline results of the Depiscan study: A French randomized pilot trial of lung cancer screening comparing low dose CT scan (LDCT) and chest X-ray (CXR). Lung Cancer. 2007;58:50-8.

12. Infante M, Chiesa G, Solomon D, Morenghi E, Passera E, Lutman FR, et al Surgical procedures in the DANTE trial, a randomized study of lung cancer early detection with spiral computed tomography: comparative analysis in the screening and control arm. J Thorac Oncol. 2011;6:327-35.

13. Pedersen JH, Ashraf H, Dirksen A, Bach K, Hansen H, Toennesen P, et al. The Danish randomized lung cancer CT screening trial-overall design and results of the prevalence round. J Thorac Oncol. 2009;4:608-14.

14. Gopal M, Abdullah SE, Grady JJ, Goodwin JS. Screening for lung cancer with low-dose computed tomography: a systematic review and meta-analysis of the baseline findings of randomized controlled trials. J Thorac Oncol. 2010;5: 1233-9.

15. National Lung Screening Trial Research Team, Aberle DR, Adams AM Berg CD, Black WC, Clapp JD, et al. Reduced lung-cancer mortality with low-dose computed tomographic screening. N Engl J Med. 2011;365:395-409.

16. Henschke CI, McCauley DI, Yankelevitz DF, Naidich DP, McGuinness G, Miettinen OS, et al. Early Lung Cancer Action Project: overall design and findings from baseline screening. Lancet. 1999;354:99-105.

17. Korst RJ, Kansler AL, Port JL, Lee PC, Altorki NK. Accuracy of surveillance computed tomography in detecting recurrent or new primary lung cancer in patients with completely resected lung cancer. Ann Thorac Surg. 2006;82: $1009-15$.

18. Lou F, Huang J, Sima CS, Dycoco J, Rusch V, Bach PB. Patterns of recurrence and second primary lung cancer in early-stage lung cancer survivors followed with routine computed tomography surveillance. J Thorac Cardiovasc Surg. 2013;145:75-82

19. Cangemi V, Volpino P, D’Andrea N, Puopolo M, Fabrizi S, Lonardo MT, et al Local and/or distant recurrences in T1-2/N0-1 non-small cell lung cancer. Eur J Cardiothorac Surg. 1995;9:473-8

20. Martini N, Bains MS, Burt ME, Zakowski MF, McCormack P, Rusch VW, et al Incidence of local recurrence and second primary tumors in resected stage I lung cancer. J Thorac Cardiovasc Surg. 1995;109:120-9.

21. Immerman SC, Vanecko RM, Fry WA, Head LR, Shields TW. Site of recurrence in patients with stages I and II carcinoma of the lung resected for cure Ann Thorac Surg. 1981;32:23-7.

22. Hamaji M, Allen MS, Cassivi SD, Deschamps C, Nichols FC, Wigle DA, et al Surgical treatment of metachronous second primary lung cancer after complete resection of non-small cell lung cancer. J Thorac Cardiovasc Surg. 2013;145: 683-91.

23. Lamont JP, Kkuda JT, Smith D, Wagman LD, Grannis FW. Systematic postoperative radiologic follow-up in patients with non-small cell lung cancer for detecting second primary lung cancer in stage IA. Arch Surg. 2002;137:935-9.

24. Mollberg NM, Ferguson MK. Postoperative surveillance for non-small cell lung cancer resected with curative intent: developing a patient-centered approach. Ann Thorac Surg. 2013;95:1112-21.

\section{Discussion}

Dr Michael T. Jaklitsch (Boston, Mass). Thank you very much. It is my privilege to discuss this remarkable paper by Dr Hanna and his colleagues at the University of Toronto on minimal-dose CT scan for screening of lung cancer recurrence in those patients who have undergone a successful surgical operation for early stage lung cancer.

Last year at this meeting the AATS task force for lung cancer screening and surveillance reported its guidelines for screening 
for new populations, but also it was our specific mandate to address what should be done for screening for surveillance of lung cancer survivors. We were struck by the paucity of hard scientific data to draw upon our guidelines and resorted to anecdotal experience of large centers that had a vast experience, including following the traditions that were established at the University of Toronto in the 1980s. I congratulate Dr Hanna and his colleagues for now providing that surgical evidence that gives meat to the specific guidelines.

Screening for early stage lung cancer with minimal harm and maximum benefit is an interaction of several variables, including the baseline risk of the screened population, the interval of screening, and the technology of screening. Dr Hanna shows that this particular population is the highest risk, in fact, a $10 \%$ risk over 5 years. That is twice the risk of what we normally deem as a high-risk subpopulation of $5 \%$ over 5 years, and the NLST data, which showed a $20 \%$ reduction in lung cancer-specific mortality, had a $2 \%$ risk over 5 years.

Furthermore, he has technology that is really impressive. Dropping that $\mathrm{mSv}$ dose down to 0.2 , so it really is comparable to chest $\mathrm{x}$-ray, eliminates the potential harms of the operation, and they combine this to now introduce the idea of surgical salvage for early stage lung cancer. So, in fact, they were able to take a group that would have had a 5-year survival of 50\% and raise it up to $75 \%$ through an aggressive surveillance program, and, furthermore, they established that they were truly curing these patients.

Is this enough data to change our personal practices today? For me personally the answer is yes. This single paper presents to me enough data to say that I want to use a minimal-dose CT scan as my sole method of screening for recurrence of early stage lung cancer moving forward. And although he states that minimal-dose CT is not available at every center in the United States, I was able to take the specifications that Dr Hanna provided me with in his paper to my community hospital, and my community hospital assures me that they can do this. So, at least in the United States of America, I think that this will have dramatic penetrance.

My specific question, Dr Hanna, is, are there other variables that come into play that may limit this technology? In particular, one of the issues is scatter of the x-ray beam. If I have a very heavyset patient who has a BMI of, say, 40, is this technology going to work or am I going to have to increase the dose? What if I have a lot of surgical clips at the hilum? Is that going to produce scatter? Do you at least have some anecdotal evidence that suggests that there is a subpopulation in which this will not work?

Thank you for the privilege of discussing this paper.

Dr Hanna. Thank you for your comments, Dr Jaklitsch, and for taking the time to discuss our paper. Your question is very important because a lot of radiologists and surgeons ask us about the accessibility of minimal dose in terms of interpretation. There are certain limitations to the minimal-dose technology. You mentioned patients of a particular body habitus and a high BMI. These are problematic because the noise on the CT scan image is usually very high and then you cannot really see small detail. So, in these patients, one would recommend a low-dose CT rather than minimal-dose CT, at least at our institution. Other limitations of minimal-dose CT would be mediastinal imaging. If you have a patient in whom you are suspecting a high recurrence rate in the mediastinum, such as a patient who had a resected tumor where there was mediastinal involvement or close margins, then our radiologists feel more comfortable with low-dose CT rather than minimal-dose CT for the surveillance of these patients. You also mentioned surgical clips in the area which contribute to the noise and make minimal-dose CT less sensitive.

Dr Bryan Fitch Meyers (St. Louis, Mo). That was an excellent paper. I am not likely to be quite as quick to adopt it as Dr Jaklitsch, but I would be happy to study the process. I have one question: with early stage (I/II) lung cancer, we expect about $20 \%$ to recur systemically, and about $2 \%$ per year to develop a new primary tumor. If your results presented today are applicable to the general population of resected lung cancer patients, it would be reassuring to know that you found the same rate of recurrence and new primaries in your patients, or did you have an enriched population by somehow selecting higher-risk patients?

Dr Hanna. Thank you. This is a very important question. As we have demonstrated, most of our patients were stage I disease. So we did find a lower rate of systemic recurrence than what we expected in a general population of all-comers. However, we do think that surveillance with minimal-dose $\mathrm{CT}$ will shift those rates of recurrence or new primary tumors. We do not make a distinction between recurrence or new primary because we do not think that in terms of surveillance it matters - these patients are at high risk for both events.

Dr Chumy Nwogu (Buffalo, NY). My question arises from your calculation of sensitivity for your minimal-dose CT. What did you use as your gold standard to do that calculation and did you have a direct comparison between the regular CT scan and your minimal-dose CT to see what the differences would be? I think that would help us tremendously in deciding whether to adopt this in our daily practices.

Dr Hanna. Thank you very much. It is a very important point. We did not have a direct comparison between standard-dose CT and minimal-dose CT, but our gold standard of determining whether a patient recurred or not was most of the time a standard-dose CT. When a minimal-dose CT study was deemed positive by the radiologist and the surgeon, the patient would go on to a standard-dose CT, and if the findings were suspicious enough for recurrence, the patient would be biopsied, restaged, or treated. So most of the time the standard-dose CT was a reference point to calculate sensitivity.

In patients who we still missed on standard-dose CT, the eventual development of metastatic disease or local recurrent disease was considered as the reference point, and in patients who were treated palliatively because of enough suspicion on imaging that they had recurrent or metastatic disease, then this was our reference point. Thank you.

Dr Frank C. Detterbeck (New Haven, Conn). I think this is a very exciting paper. I am struck, though, that your incidence of finding a lesion early is quite high, and there are many institutions that have done CT scans every 6 months or every year. I am sure you have done that in the past. Have you compared this with either your prior results or other institutional results at a year, because those that were found at 3, 6, 9, and 12 months should have shown up at the yearly scan.

Dr Hanna. Thank you very much. We still do not know exactly the ideal interval for obtaining scans. Is it 3 months or 6 months or a 
year? We decided on the 3-month and 6-month intervals because it is part of the international consensus statement, and I do agree that some of the lesions that we found at 3 months or 6 months could have been found at a year if we did a yearly scan without much difference in outcome. So this remains to be studied.

But I do think it is also important to recognize that now there is a shift in terms of when we diagnose recurrence or new primary after lung cancer, and it is going to be earlier and earlier.

Dr David J. Sugarbaker (Boston, Mass). Just a quick question, and that is, what you have really described is the detection of new primary tumors following initial resection. Particularly in $\mathrm{T} 2$ tumors, the 2 sites of recurrence where long-term therapy is usually not associated with prolonged survival are, as you said, mediastinal node disease and distant metastatic disease. So I think it is important to realize that what you are really talking about here is screening patients who have had lobar resections or any resections for lung cancer.
So I am not sure your conclusion about follow-up detection of metastatic disease is really an accurate description as to what you have done. And, particularly with the inability of minimal-dose CT to assess the mediastinum, particularly in the larger lesions where distant metastatic disease may be more frequent, I am not sure that minimal dose CT is adequate for those patients where, again, mediastinal nodal disease can be the real issue.

Could you comment?

Dr Hanna. Thank you. I do agree with that. I think minimal-dose CT is a good test for detection of a new primary. However, we realize that these patients are also at risk for mediastinal disease, and I think the future will hold a more patient-centered approach to follow-up whereby patients with a higher risk of distant recurrence will have some other tests on top of the minimal-dose CT to try and detect that earlier. 